\title{
BMJ Open Incorporating patient-reported outcomes into shared decision-making in the management of patients with osteoarthritis of the knee: a hybrid effectiveness-implementation study protocol
}

\author{
Eugenia Lin, ${ }^{1}$ Lauren M Uhler (10 , ${ }^{1}$ Erin P Finley, ${ }^{2,3,4}$ Prakash Jayakumar, ${ }^{1}$ \\ Paul J Rathouz, ${ }^{5}$ Kevin J Bozic, ${ }^{1}$ Joel Tsevat ${ }^{3,4,5}$
}

To cite: Lin E, Uhler LM, Finley EP, et al. Incorporating patient-reported outcomes into shared decision-making in the management of patients with osteoarthritis of the knee: a hybrid effectiveness-implementation study protocol. BMJ Open 2022;12:e055933. doi:10.1136/ bmjopen-2021-055933

- Prepublication history and additional supplemental material for this paper are available online. To view these files, please visit the journal online (http://dx.doi.org/10.1136/ bmjopen-2021-055933).

Received 03 August 2021 Accepted 20 January 2022

Check for updates

(c) Author(s) (or their employer(s)) 2022. Re-use permitted under CC BY-NC. No commercial re-use. See rights and permissions. Published by BMJ.

For numbered affiliations see end of article.

Correspondence to

Dr Joel Tsevat;

tsevat@uthscsa.edu

\begin{abstract}
Introduction 0steoarthritis $(O A)$ is a major clinical and public health concern. The primary surgical treatment of knee $O A$ is total knee replacement (TKR), a procedure that aims to alleviate pain and restore physical function. TKR is expensive, however, and based on professional guidelines, inappropriately performed in up to a third of patients. Patient-reported outcome measures (PROMs) help evaluate treatment options by quantifying health outcomes that matter to patients and can thus inform shared decision-making (SDM) between patients and health professionals.
\end{abstract}

Methods and analysis This is a US-based 2-year, twosite hybrid type 1 study to assess clinical effectiveness and implementation of a machine learning-based patient decision aid (PDA) integrating patient-reported outcomes and clinical variables to support SDM for patients with knee OA considering TKR. Substudy 1: At one study site, a randomised controlled trial is evaluating the clinical effectiveness of the PDA and SDM process on decision quality as measured after the baseline consultation and treatment choice measured 3 and 6 months after the baseline visit among 200 patients with knee 0A. Substudy 2: At a second study site, a qualitative assessment using principles of behaviour design and intervention mapping is evaluating the feasibility and acceptability of the PROMs, PDA and SDM process by interviewing seven health professionals and 25 patients before and 25 patients after PDA implementation.

Ethics and dissemination Ethics approval has been obtained from The University of Texas at Austin Institutional Review Board (protocol number: 2018-11-0042). Informed consent will be obtained from all participants. Study results will be disseminated through conference presentations, publications and professional societies.

Trial registration number NCT04805554.

\section{INTRODUCTION}

Osteoarthritis (OA) of the knee constitutes a major clinical and public health problem. ${ }^{1}$
Strengths and limitations of this study

- A key study design strength is the use of hybrid effectiveness-implementation methods and principles of behaviour design and implementation mapping.

- A machine learning-based tool has a theoretical advantage over a static patient decision aid by continuously refining its prediction algorithms with new input data.

- Another strength is conducting the study at two orthopaedic surgery practices with different patient populations, clinical team configurations and electronic health record systems.

- The primary limitation of this study is the generalisability of findings to other sites.

This common and disabling condition has a substantial detrimental impact on affected individuals and society at large, accounting for over $\$ 27$ billion in US healthcare costs annually. ${ }^{2}$ Treatment options for knee OA range from lifestyle changes to pharmacological management to total knee replacement (TKR) surgery. While TKR has a strong track record in alleviating pain and improving functional limitations in individuals with advanced knee OA, there are growing concerns over the escalating volume and cost of these procedures. TKR is one of the most common elective surgical procedures: the estimated number of people living in the USA in 2010 who have had a TKR was 4.7 million, with widespread variation in rates across states. By 2030, 7.4 million are expected to have knee replacement. ${ }^{3}$ Thus, appropriate application of TKR for the right patient at the right time is critical, especially 
within existing fee-for-service structures that incentivise performing more procedures. ${ }^{4-8}$ Notably, up to $33 \%$ of TKRs have been shown to be inappropriate based on criteria developed by the American Academy of Orthopaedic Surgeons, resulting in a substantial proportion of patients failing to experience improvement in the outcomes that matter to them. ${ }^{9}{ }^{10}$ Such outcomes can be captured using patient-reported outcome measures (PROMs) - surveys that score aspects of a person's physical, psychological and social health and well-being, directly from their perspective without interpretation by a clinician or researcher. ${ }^{11}$ PROMs have now been used extensively in clinical research to evaluate health status and are increasingly being applied in clinical care to monitor health outcomes and support shared decisionmaking (SDM).

\section{SDM, patient decision aids and patient-reported outcomes}

SDM is a "process of communication in which clinicians and patients work together to make informed health care decisions that align with what matters most to patients' ${ }^{12}$ SDM and active patient participation in decision-making can be facilitated by patient decision aids (PDAs) - tools that can help people make informed decisions through patient education, knowledge assessment, elicitation of patient preferences and decision support. ${ }^{13} \mathrm{SDM}$ is most appropriate for 'preference-sensitive' conditions, such as $\mathrm{OA}$ of the knee, where multiple treatment options exist and the patient preferences and values are critical in making informed treatment choices. Thus, making a decision to undergo TKR should incorporate SDM and understanding of patient preferences, values and goals, rather than objective clinical findings alone. The importance of SDM has been recognised at a national level by the US Centers for Medicare \& Medicaid Services (CMS), which ties the concept to coverage of certain other interventions including lung cancer screening and two cardiac procedures. ${ }^{14}$ CMS and other payers are also promoting the use of PROMs within contemporary alternative payment arrangements such as the Comprehensive Care for Joint Replacement Model-a mandatory bundled payment programme for 67 geographic areas that includes a quality incentive for submitting patientreported outcomes (PROs), as measured by PROMs. ${ }^{15}$

Administering PROMs and performing SDM at the point of care have been well studied separately, ${ }^{16-20}$ and guidelines on implementing $\mathrm{SDM}^{21}$ and best practices for collecting and using PROs ${ }^{22}$ have been published extensively. Recent work to incorporate PROMs into clinical decision-making includes a project funded by the US Agency for Healthcare Research and Quality assessing patient and clinician preferences, understanding, usability and acceptability of PRO score visualisation and presentation in patient portals and electronic health records (EHRs) $;^{23}$ a project creating and evaluating a learning network in public hospital systems to increase the use of PROMs in rheumatoid arthritis and create scalable natural language processing systems to extract PROs from clinical notes; ${ }^{24}$ and a project looking at ways to integrate 'patient-preferred' hip and knee PRO scores into the EHR for use at the point of care.$^{25}$ PDAs are generally static in the sense that their calculations are not updated with new input data. A machine learning-based tool has a theoretical advantage over a static PDA by continuously refining its prediction algorithms with new input data. Still, studies evaluating the impact of a PRO-driven, machine learning technology-enabled PDA in SDM in patients with knee OA considering TKR are lacking.

In this study, we are evaluating and implementing a tool to guide SDM in two ambulatory orthopaedic surgery practices with different patient populations, levels of experience with PROMs and SDM, care delivery models and EHR systems. Specifically, the project involves integrating PROs and clinical data within a machine learning-based predictive analytical model, then using its output as part of SDM. Knowledge gained will be critical to scaling the use of PROMs and tools (PDAs) for SDM among patients with knee OA considering surgery.

We have designed a 2-year, two-site study using a hybrid type 1 study design to assess both clinical effectiveness and implementation. ${ }^{26}$ Specifically, our two aims are:

Substudy 1: In a randomised controlled trial (RCT) at one site, to evaluate the clinical effectiveness of the PRO-guided predictive analytical tool and process in terms of decision quality and treatment choice for patients with knee OA.

Substudy 2: In a qualitative assessment at the second site, to implement and evaluate the feasibility and acceptability of the tool and SDM process in a clinical setting with a different clinical population, provider group and EHR by using principles of behaviour design and intervention mapping.

\section{METHODS AND ANALYSIS}

\section{Research strategy}

Overview

This hybrid effectiveness-implementation study includes a non-blinded RCT of effectiveness outcomes at one site, plus periodic reflections and semistructured interviews with providers and patients to evaluate implementation processes and outcomes (eg, feasibility and acceptability) at a second site. Data will be integrated following recommended principles for mixed methods research to inform ongoing refinements to the predictive analytical tool (via formative evaluation) ${ }^{27}$ and plans for scaling (via intervention mapping). ${ }^{2829}$

Joint Insights (artificial intelligence-enabled SDM tool) and PROMs Joint Insights (OM1, Boston, Massachusetts) is a machine learning-enabled PDA that uses PROMs along with patient clinical and demographic information (age, sex, body mass index, smoking status, comorbidities and number of times the patient has recently visited an emergency department or has been hospitalised) to provide 

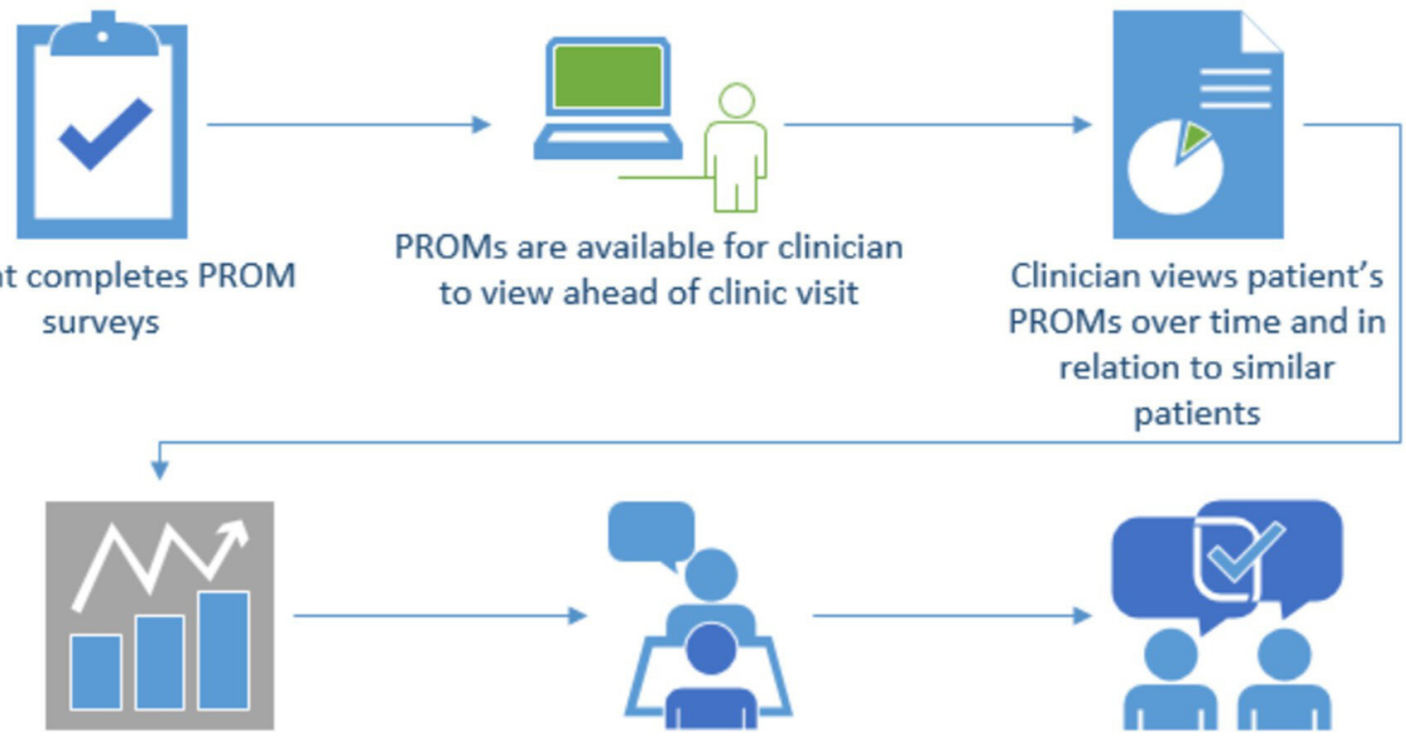

\section{Algorithm calculates likelihood of patient achieving meaningful improvement with surgery}

Clinician and patient discuss the patient's likely benefits and risks from surgery

\section{Patient and clinician jointly arrive} at a treatment decision

Figure 1 Process flow of patient-reported outcome measures (PROM) within the clinical pathway.

personalised estimates of likely benefit or harm from TKR (figure 1). ${ }^{27}$ The tool is designed to collect PROs or pull in PROs collected through other systems (eg, an EHR or a third-party PROM platform). It also provides conditionspecific education to patients with knee $\mathrm{OA}$ and allows a patient to reflect on and document their preferences and goals. Patient journeys are drawn from the OM1 Intelligent Data Cloud for patients undergoing TKR who have adequate follow-up for the outcome being evaluated. Approximately 675000 patient records were used for the original risk model, which continues to be updated. In the modelling population (risk model), $60.8 \%$ of patients are male, the mean age is 65 years and the mean body mass index is $31.8 \mathrm{~kg} / \mathrm{m}^{2}$. The PROMs used with Joint Insights include the Patient-Reported Outcomes Measurement Information System (PROMIS) Global-10 physical and mental health subscores ${ }^{30}$ and the Knee injury and Osteoarthritis Outcome Score for Joint Replacement (KOOS JR). ${ }^{31}$ PROMIS subscores are expressed as t-scores, with 50 representing the population mean; higher scores indicate better physical function (physical function subscore) but worse mental health (mental health subscore). The KOOS JR is a seven-item PROM encompassing questions on function, pain and stiffness and scored using a scale from 0 to 100 , where 0 represents poorest knee health and 100 represents best knee health.

\section{Study dates and sites}

This is a 2-year study planned from September 2020 to August 2022. The recruitment start date of this study was 22 February 2021. The PDA has already been integrated into the workflow of the UT Health Austin Clinic, where the effectiveness trial (substudy 1) is taking place. The study design and choice of different setting (UT Health
San Antonio) for the implementation study (substudy 2) is intended to elucidate the feasibility and acceptability of implementing the tool into a clinic with a different population; care delivery team having less familiarity with using PROs routinely in practice; and a different EHR system, which automatically uploads PRO scores for the clinician to view at the point of care (table 1$)$.

\section{Substudy 1 overview}

Substudy 1 is projected to take place in year 1 and early in year 2 at the UT Health Austin Musculoskeletal Institute in Austin, Texas. Patients are randomised to one of two arms: intervention, with the full Joint Insights tool

\begin{tabular}{|c|c|c|}
\hline & $\begin{array}{l}\text { UT Health Austin } \\
\text { (Substudy 1) }\end{array}$ & $\begin{array}{l}\text { UT Health San Antonio } \\
\text { (Substudy 2) }\end{array}$ \\
\hline $\begin{array}{l}\text { Patient } \\
\text { population }\end{array}$ & $\begin{array}{l}50 \% \text { MAP patients } \\
32 \% \text { Spanish primary language }\end{array}$ & $\begin{array}{l}2 \% \text { uninsured } \\
12 \% \text { Spanish primary } \\
\text { language }\end{array}$ \\
\hline Care team & $\begin{array}{l}\text { Orthopaedic surgeons } \\
\text { Associate providers (NPs) } \\
\text { Social worker, dietician }\end{array}$ & $\begin{array}{l}\text { Orthopaedic surgeon } \\
\text { Associate provider (NP) }\end{array}$ \\
\hline EHR & Athena & Epic \\
\hline $\begin{array}{l}\text { PRO collection } \\
\text { methods }\end{array}$ & $\begin{array}{l}\text { Clinect (email previsit), tablet- } \\
\text { based collection as backup }\end{array}$ & $\begin{array}{l}\text { Epic MyChart portal } \\
\text { (previsit), tablet-based } \\
\text { collection as backup }\end{array}$ \\
\hline $\begin{array}{l}\text { PRO collection } \\
\text { uptake }\end{array}$ & 100\% of patients & Limited \\
\hline PROs collected & $\begin{array}{l}\text { General health } \\
\text { Mental health (depression, } \\
\text { anxiety) } \\
\text { Hip and knee specific }\end{array}$ & \\
\hline
\end{tabular}

EHR, electronic health record; MAP, Medical Access Program (covers healthcare for otherwise uninsured patients in Travis County); NP, nurse practitioner; PRO, patientreported outcome. 
(including education on knee OA and treatment options, preference elicitation questions and personalised benefit/risk report), or control, receiving only the educational component of the tool and preference elicitation questions. The enrolment target is 180 , but to account for loss to follow-up, we are enrolling 200 patients. Quantitative outcomes include the primary endpoint-decision quality - as assessed at the conclusion of the initial consultation by using the previously validated decision process subscore of the Decision Quality Index (DQI) for knee $\mathrm{OA},{ }^{32}$ and, as secondary endpoints, level of SDM from the patient's perspective (CollaboRATE) ${ }^{33}$ aspects of decision conflict (Decision Conflict Scale 10 (DCS-10)) $;^{34} 35$ and decision regret (Decision Regret Scale (DRS) ). ${ }^{36}$ The DQI, CollaboRATE and DCS-10 will be assessed at the end of the baseline visit, and the DRS will be assessed at 3 and 6-month follow-up visits (or by phone or email if patients do not return to the clinic for a visit). As an additional endpoint, we will capture the OA treatment selected (operative vs non-operative), assessed at the 3 and 6-month follow-up.

\section{Substudy 2 overview}

Substudy 2 is being carried out over both years primarily at UT Health San Antonio, Texas. Year 1 has entailed preparing UT Health San Antonio's EHR to collect PROMs, preparing the EHR for integration of the predictive analytical tool, assessing baseline feasibility and acceptability and working with the clinic site to develop an implementation plan. Baseline interviews conducted with San Antonio providers and staff inquired about acceptability and feasibility of collecting PROs and using the tool, as well as exploring key factors (barriers and facilitators) impacting motivation and ability to implement the tool and SDM process at the individual and clinic levels. Interview guides were tailored to clinical role (eg, surgeon, resident, staff) and reflected implementation concepts based on the Consolidated Framework for Implementation Research (CFIR) and behaviour design, which theorises that any given behaviour is most likely to manifest when motivation, ability and a prompt to carry out the behaviour all occur in the same moment (see online supplemental information). ${ }^{37}$ In collaboration with the site's clinical team, we are identifying each step in the workflow necessary to collect PROMs, incorporate the PRO scores and clinical variables into Joint Insights and conduct an SDM consultation for a single patient, and assessing team perspectives on the barriers and facilitators of each step in this workflow being achieved. These data then go into developing a preliminary plan for implementation at the site, which in turn is refined iteratively in collaboration with the clinical team. Post implementation interviews are planned with providers and staff 3 months following tool roll-out to assess reported use of and experiences with the PROMs and Joint Insights tool, adaptations to tool use and workflow integration and factors impacting the likelihood of sustainment of the process of care.
Semistructured interviews have also been conducted with 25 patients prior to implementing PROMs and Joint Insights in order to assess: priorities and hopes for treatment (eg, CFIR: patient needs and resources); experience of discussing treatment options with providers; and expectations for next steps in their treatment process. Then, 3 months following implementation, 25 new patients will be interviewed to assess the experiences with and acceptability of the Joint Insights tool.

Finally, periodic reflections are being conducted with members of the Austin and San Antonio implementation teams in order to document implementation processes, adaptations and contextual factors at each site. Periodic reflections are an established, low-burden method for capturing dynamic factors affecting implementation of health interventions. ${ }^{38}$

\section{Substudy 1}

Practice settings, patient populations and use of PROs: UT Health Austin Musculoskeletal Institute

The UT Health Austin Musculoskeletal Institute averages about 12 new patients presenting with knee OA per week. Patients are seen by a care team that may include an associate provider (nurse practitioner), physical therapist, social worker, nutritionist and/or surgeon depending on the patient's needs. Approximately $60 \%$ of patients are women; $50 \%$ are uninsured but covered by the Medical Access Program (MAP), which provides access to care for uninsured low-income residents of Central Texas; and $32 \%$ speak Spanish as their primary language. Musculoskeletal providers collect general and condition-specific PROs from every patient seen in the Musculoskeletal Institute (figure 2). The practice has experience with PROMs, the Joint Insights tool and SDM. PROs are collected for clinical purposes via an electronic interface and results are pulled into the EHR (Athena, Watertown, Massachusetts). Investigators (KJB, PJ) from UT Austin worked with OM1 to codevelop the PDA.

\section{Participant selection}

\section{Inclusion criteria}

- New patients aged 45-89 with a presumptive diagnosis of knee OA.

- Kellgren-Lawrence Scale joint space narrowing grade 3 or 4 (moderate to severe OA) and KOOS JR scores between 0 and 85 .

- Ability to give informed consent for participation in the study.

- Ability to read text at the eighth grade reading level on a tablet in English or Spanish.

\section{Exclusion criteria}

- Patients with a prior TKR or prior consultation with another orthopaedic surgeon for TKR.

- Patients having prior experience with the Joint Insights tool.

- Patients undergoing consideration for revision joint replacement. 


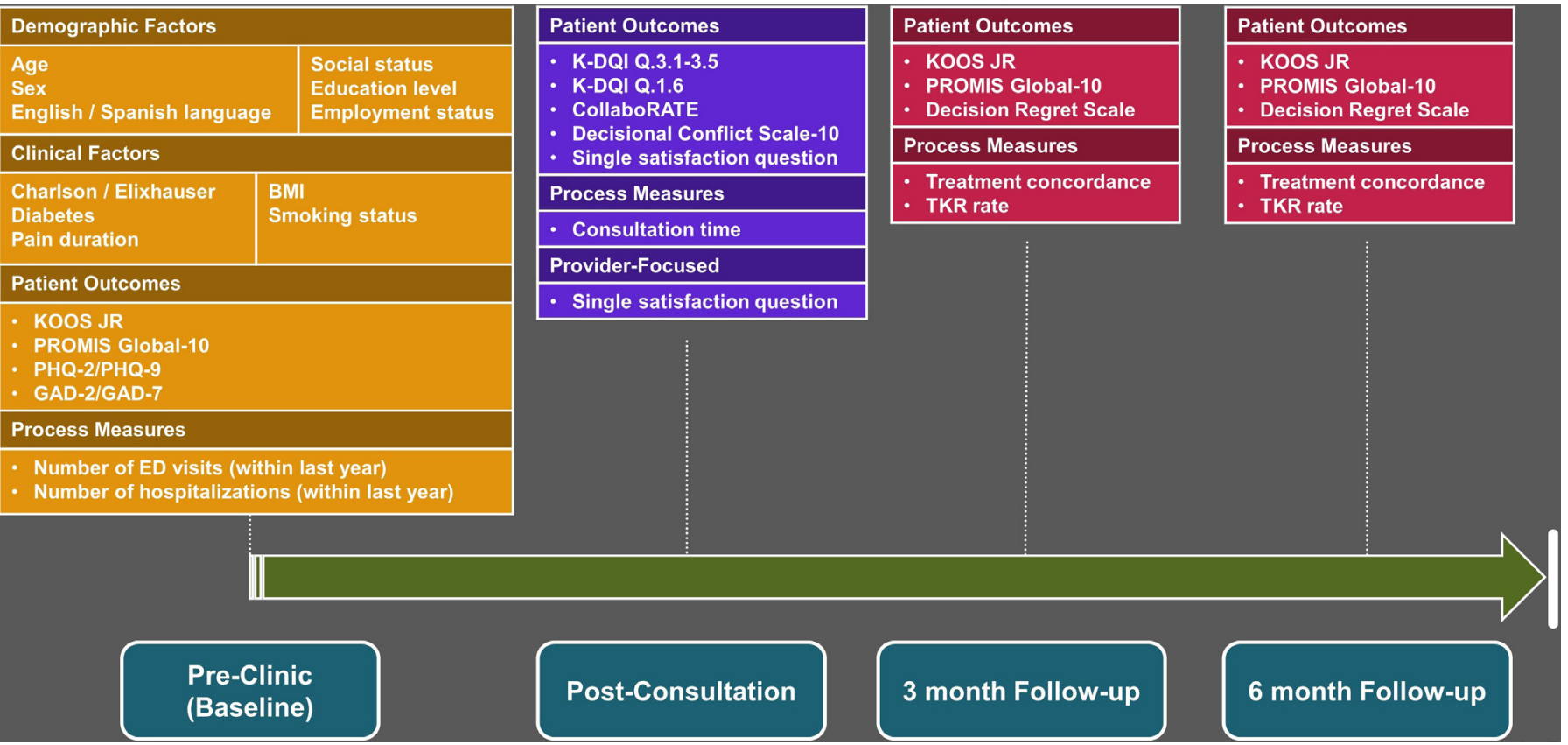

Figure 2 Outcomes collected at UT Health Austin. The Knee injury and Osteoarthritis Outcome Score for Joint Replacement (KOOS JR) is a seven-item patient-reported outcome measure of knee joint-related stiffness, pain and function; interval scores range from 0 to 100, with 0 representing poorest knee health and 100 best knee health. The Patient-Reported Outcomes Measurement Information System (PROMIS) Global-10 is a 10-item measure assessing health-related quality of life with items about overall physical and mental health including social connections and physical capabilities. The survey is scored using two subscores, one for physical health and one for mental health, where specific items are used for a raw score and then converted to a t-score. Population norm t-scores are 50 on each subscore; higher scores reflect better physical health but worse mental health. The Patient Health Questionnaire $(\mathrm{PHQ})^{44} 45$ is a validated two-item or nine-item survey assessing depressive symptoms and scored categorically as none, mild, moderate, moderately severe and severe. The two-item questionnaire is deployed, and if crossing a score threshold, an additional seven questions are generated. The Generalized Anxiety Disorder Questionnaire $(G A D)^{46} 47$ is a two-item or seven-item survey assessing generalised anxiety disorder and scored categorically as none, mild, moderate and severe. Similar to the PHQ, if a score threshold is crossed on the two-item form, an additional five questions are generated. The Knee Decision Quality Instrument (K-DQI) is a 16-item survey with three specific scores: a total knowledge score, a concordance score and a decision process score. For the purposes of this study, the five questions in the shared decision-making section are used. One point is scored for 'yes' or 'a lot/some'. These points are summed and then divided by 5 , resulting in a score from $0 \%$ to $100 \%$, with higher scores indicating a greater level of shared decision-making. The CollaboRATE is a three-item, 10-point anchor scale measuring the level of shared decision-making in a clinical encounter. It yields a continuous score with a possible range from 0 to 100, where higher scores represent a greater degree of shared decision-making. The Decision Conflict Scale (DCS) is a 10-item survey, with each response value summed, divided by the total item number and multiplied by 25. The score ranges from 0 to 100, where 0 is no decisional conflict and 100 is the greatest decisional conflict. Finally, the Decision Regret Scale (DRS) measures distress or remorse after making a healthcare decision. The answer values are summed and converted to a 0-100 scale, where a higher score indicates more regret. BMI, body mass index; ED, emergency department; TKR, total knee replacement.

- Patients seeking care for trauma, psoriatic arthritis or rheumatoid arthritis.

- Patients with a body mass index less than $20 \mathrm{~kg} / \mathrm{m}^{2}$ or greater than $46 \mathrm{~kg} / \mathrm{m}^{2}$.

\section{Participant recruitment and data collection}

The UT Health Austin Musculoskeletal Institute sees a mix of patients seeking care for knee OA comprising a range of pathological severity - individuals who are referred from primary or specialty care or are selfreferred. Suitable patients for the study are identified during the preclinic meeting. Once the patient has entered the clinic room or private consultation space, they are met by a researcher and invited to participate in the study. If they agree to participate, the researcher obtains informed consent. We are using the randomisation module of REDCap, a HIPAA-compliant research database. We are stratifying patients who enroll in the RCT on three variables: ethnicity (Latino/non-Latino), insurance (MAP/non-MAP) and orthopaedist seen (provider A (author KJB) vs provider B). This stratification ensures balance of these three variables between intervention and control groups over time and within stratum. Patients from each of the resulting eight strata are randomised to intervention or control in randomly sequenced blocks of four or six. Neither provider nor study participant will know the next allocation in the sequence until the participant is consented and it is time to begin the intervention. Due to the nature of the 
intervention, patients, researchers and clinicians are not blinded to treatment arm assignment.

Demographic information is collected via tablets after randomisation. Next, patients in the intervention group receive a Joint Insights risk/benefit report. Those randomised to the intervention group may review and discuss the Joint Insights report as part of the clinical visit. The control group does not receive the Joint Insights report. Following the completion of the visit, survey instruments are collected for participants in both arms by using REDCap forms on the tablet. At 3 and 6 months of follow-up, participants are given follow-up surveys on REDCap either in person, by email or by phone. Participants completing follow-up surveys receive a $\$ 25$ gift card.

\section{Statistical precision and sample size}

We calculated the sample size for the RCT by treating the decision process score of the DQI as continuous. We aimed to detect a treatment effect size (ie, Cohen's $D$ ) as small as 0.5 (consistent with preliminary data from the first 26 subjects we have studied) with a type I error rate of 0.05 and power of 0.90 , assuming equal sample size in intervention and control groups. Given our eight randomisation strata, we estimate a needed sample size of 180 participants, or 90 for each group. With an estimated loss to follow-up rate of $10 \%$, our target enrolment for the RCT is 200 participants, or 100 for each arm.

\section{Quantitative analysis}

For the RCT in Austin, formal comparative analysis will follow the intent-to-treat principle. Primary analysis will compare the intervention and control groups by using multiple linear regression analysis. The model will include DQI score as the response variable and, as explanatory variables, a binary indicator for the intervention group and seven binary indicator variables representing the eight strata in order to reflect the stratified randomisation design. Additionally, as a secondary analysis, we will compare treatment decisions between the intervention and control groups by using multiple logistic regression. The model will include the treatment decision as the binary response variable and the same explanatory variables as in the linear regression model. Depending on the uptake of the intervention, additional analyses will follow the per-protocol principle where the main treatment variable will be whether the Joint Insights tool was actually used.

For analysis of the 3- and 6-month data, we will fit the linear mixed models for continuous outcomes ${ }^{39}$ and generalised estimating equations logistic regression models for binary outcomes,${ }^{40}$ including indicator variables for time point, for treatment group and for the interaction between the two (yielding treatment effects at 3 and 6 months). Owing to the balanced design, it will be possible to fit an unstructured correlation model to eliminate any sensitivity to correlation model misspecification.

\section{Substudy 2}

Practice settings, patient populations and use of PROs: UT Heath San Antonio Medical Arts \& Research Center

This academic practice in San Antonio currently has one orthopaedist who treats the vast majority of patients with knee OA. This provider and a care team comprising resident physicians and an associate provider (nurse practitioner) see 16-26 new patients with knee OA per week, in addition to returning patients with OA. As in Austin, approximately $61 \%$ of patients are women, but in contrast to Austin, only $2 \%$ are uninsured and $12 \%$ report that Spanish is their primary language. The clinic had not implemented PRO collection prior to this study. The clinic uses Epic (Epic, Verona, Wisconsin) as its EHR. PROs are collected either through Epic's MyChart patient portal in advance of the patient's appointment or via tablets in the clinic on the day of the appointment. PRO scores are then transmitted to clinicians through the EHR's clinician interface.

\section{Participant recruitment}

The Medical Arts \& Research Center Orthopaedics Clinic in San Antonio sees a mix of patients seeking care for knee OA or considering TKR, and a mix of patients who are referred or self-referred. New patients being seen for possible TKR are contacted by project staff to schedule an interview to be conducted either in person immediately following their clinic appointment or by Zoom within the subsequent 1-2 days. A research associate obtains informed consent from all willing patients; participants who complete an interview receive a $\$ 25$ gift card as compensation.

\section{Sample size calculation}

For staff and provider interviews, we have invited every member of the clinical team to participate in order to have full representation of those involved in implementation. In developing our patient sample, we considered the need to capture heterogeneity in patient demographics, condition severity, need for surgery, health literacy and preferences for treatment planning, while also acknowledging the relative homogeneity of the patient population being evaluated for knee replacement surgery in a single orthopaedic clinic. Following recommendations for ensuring information power, as specified by Malterud and colleagues, ${ }^{41}$ we estimated that a sample of 25 patients at each time point would provide adequate information power to represent a broad range of patient experiences and perspectives.

\section{Qualitative analysis}

All interviews are audio recorded for transcription and analysis. Interview data will be analysed using established processes for rapid qualitative analysis. ${ }^{42}$ We will create structured summaries from transcribed recordings to capture key domains drawn from CFIR, behaviour design and emerging content reflecting provider, staff and patient perspectives. We will then transpose domain 
content from summaries into a matrix to allow for structured content comparison across participants and domains (ie, matrix analysis), an effective method for rapid and rigorous summary of findings to aid formative and implementation evaluation. ${ }^{43}$ In accordance with behaviour design and intervention mapping, we will then identify key factors impacting motivation and ability across each CFIR construct identified, separating out by stakeholder group (clinic staff, providers). For example, Joint Insights-based SDM may be perceived to be relatively advantageous (CFIR domain: intervention characteristics) by comparison with previous practice but may also raise concerns about staff burden. We will create a visual map to summarise staff and provider suggestions and concerns across each step of the Joint Insights tool implementation workflow; this map will aid collaborative implementation planning. Data from periodic reflections will also be analysed by using rapid qualitative methods in order to assess key events occurring during implementation (eg, adaptations) and factors impacting implementation (eg, barriers and facilitators). These findings will be used to support scale-up and spread of Joint Insights-based SDM and the collaboratively developed implementation strategy in future research, should results of substudy 1 suggest that the intervention is clinically beneficial.

\section{Patient and public involvement}

Patients and industry stakeholders assisted with design and feedback of the Joint Insights PDA tool for readability and usability prior to the start of this research study. Specifically, the tool was shown to patients with knee pain in the UT Health Austin Musculoskeletal Institute Lower Extremity Clinic and patients were asked a short set of open-ended questions in response to viewing the riskbenefit calculator in order to assess their understanding of the information presented and their preferences for how the information was displayed. Otherwise, no formal patient or public input was involved in designing or planning this study.

\section{EXPECTED RESULTS}

Substudy 1: We expect that patients who use the full Joint Insights tool will have higher decision process scores, reflecting better decision quality, compared with those who receive the education and preference modules only. We also expect patients in the intervention group to report higher levels of SDM and lower levels of decision conflict and decision regret. We do not expect a difference in rates of treatment selected (operative vs nonoperative) between the two groups.

Substudy 2 is exploratory and therefore has no formal hypotheses.

\section{ETHICS AND DISSEMINATION}

The University of Texas at Austin Dell Medical School Institutional Review Board (IRB) reviewed and approved this study (protocol number: 2018-11-0042). The University of Texas Health Science Center at San Antonio's IRB has a formal reliance agreement with The University of Texas at Austin IRB. Any modifications to the protocol will be submitted to the UT Austin IRB for approval before implementation.

Patients and clinic staff are enrolled in this study after providing informed consent. During this study, participants complete the questionnaires related to their decision-making process and experience or are interviewed formally about their experiences. Data are kept in strict confidence. No information will be given to anyone without permission from the participant. Confidentiality is assured by use of identification codes, passwordprotected electronic files on secure servers or hosting applications and paper files stored under lock and key. The assessments are conducted in a private setting, through encrypted email or by telephone. Although we do not anticipate any adverse events, any adverse events will be reported to the local IRB.

\section{Dissemination of results}

The project will facilitate developing a learning healthcare system. PRO data will be collected electronically and used to inform clinical decision-making in real time. We will evaluate PRO data to improve clinical decisionmaking and patient outcomes locally at two sites. We will disseminate results through publications, meeting presentations and professional organisations.

\section{Author affiliations}

${ }^{1}$ Surgery and Perioperative Care, The University of Texas at Austin Dell Medical School, Austin, Texas, USA

${ }^{2}$ Research Service, South Texas Veterans Health Care System, San Antonio, Texas, USA

${ }^{3}$ Center for Research to Advance Community Health, Joe R. and Teresa Lozano Long School of Medicine, University of Texas Health Science Center at San Antonio, San Antonio, Texas, USA

${ }^{4}$ Division of General and Hospital Medicine, Department of Medicine, Joe R. and Teresa Lozano Long School of Medicine, University of Texas Health Science Center at San Antonio, San Antonio, Texas, USA

${ }^{5}$ Population Health, The University of Texas at Austin Dell Medical School, Austin, Texas, USA

Acknowledgements The authors acknowledge 0M1 as a codeveloper of the Joint Insights tool.

Contributors EL, EPF, LMU, PJ and JT wrote and edited the manuscript. EPF, LMU, $\mathrm{KJB}, \mathrm{PJR}$ and JT made substantial contributions to the conception, rationale and design of this study. EL, LMU, PJ and KJB have contributed to the intervention used in the first aim of the study. EPF and JT have significantly contributed to the design and methodology of the second aim of this study. All authors have given approval for this manuscript to be published.

Funding This project was supported by the Agency for Healthcare Research and Quality (grant number R21HS027037). PJR's effort on this project was partially supported by the core funds of Dell Medical School at The University of Texas at Austin.

Disclaimer The content is solely the responsibility of the authors and does not necessarily represent the official views of the Agency for Healthcare Research and Quality. This funding body has no direct role in the design of the study, collection, analysis or interpretation of the data.

Competing interests PJ has received personal fees from Johnson \& Johnson Medical Devices. KJB has received personal fees from the CMS and Purchaser 
Business Group on Health; has stock options from Carrum Health; and has a leadership role with the American Academy of Orthopaedic Surgeons. KJB has royalty agreements with Wolters Kluwer and Slack. KJB and PJ are codevelopers of the Joint Insights tool; they have no personal financial interest in the tool. JT receives royalties from Wolters Kluwer. The University of Texas at Austin has a royalty agreement with $0 \mathrm{M} 1$

Patient consent for publication Not applicable.

Provenance and peer review Not commissioned; externally peer reviewed.

Supplemental material This content has been supplied by the author(s). It has not been vetted by BMJ Publishing Group Limited (BMJ) and may not have been peer-reviewed. Any opinions or recommendations discussed are solely those of the author(s) and are not endorsed by BMJ. BMJ disclaims all liability and responsibility arising from any reliance placed on the content. Where the content includes any translated material, BMJ does not warrant the accuracy and reliability of the translations (including but not limited to local regulations, clinical guidelines, terminology, drug names and drug dosages), and is not responsible for any error and/or omissions arising from translation and adaptation or otherwise.

Open access This is an open access article distributed in accordance with the Creative Commons Attribution Non Commercial (CC BY-NC 4.0) license, which permits others to distribute, remix, adapt, build upon this work non-commercially, and license their derivative works on different terms, provided the original work is properly cited, appropriate credit is given, any changes made indicated, and the use is non-commercial. See: http://creativecommons.org/licenses/by-nc/4.0/.

\section{ORCID iD}

Lauren M Uhler http://orcid.org/0000-0001-6485-8207

\section{REFERENCES}

1 Centers for Disease Control and Prevention. A national public health agenda for osteoarthritis 2020 update, 2020. Available: https://www. cdc.gov/arthritis/docs/oaagenda2020.pdf

2 Losina E, Paltiel AD, Weinstein AM, et al. Lifetime medical costs of knee osteoarthritis management in the United States: impact of extending indications for total knee arthroplasty. Arthritis Care Res 2015;67:203-15.

3 Maradit Kremers H, Larson DR, Crowson CS, et al. Prevalence of total hip and knee replacement in the United States. J Bone Joint Surg Am 2015:97:1386-97.

4 Navathe AS, Liao JM, Polsky D, et al. Comparison of hospitals participating in Medicare's voluntary and mandatory orthopedic bundle programs. Health Aff 2018;37:854-63.

5 Navathe AS, Troxel AB, Liao JM, et al. Cost of joint replacement using bundled payment models. JAMA Intern Med 2017;177:214-22

6 Barnett ML, Wilcock A, McWilliams JM, et al. Two-Year evaluation of mandatory bundled payments for joint replacement. $N$ Engl J Med 2019;380:252-62.

7 Navathe AS, Liao JM, Emanuel EJ. Potential unintended effects of Medicare's bundled payments for care improvement Program-Reply. JAMA 2019;321:107.

8 Navathe AS, Liao JM, Dykstra SE, et al. Association of hospital participation in a Medicare bundled payment program with volume and case mix of lower extremity joint replacement episodes. JAMA 2018;320:901.

9 Riddle DL, Jiranek WA, Hayes CW. Use of a validated algorithm to judge the appropriateness of total knee arthroplasty in the United States: a multicenter longitudinal cohort study. Arthritis Rheumatol 2014;66:2134-43.

10 American Academy of Orthopaedic Surgeons. Quality Programs \& Guidelines (CPGS): American Academy of Orthopaedic Surgeons. Available: https://www.aaos.org/auc/?ssopc=1\#

11 Center for Drug Evaluation and Research. Patient-Reported Outcome Measures: Use in Medical Product Development [Internet]. U.S. Food and Drug Administration, 2009. Available: https://www.fda. gov/regulatory-information/search-fda-guidance-documents/patientreported-outcome-measures-use-medical-product-developmentsupport-labeling-claims

12 Barry MJ, Edgman-Levitan S, Sepucha K. Shared decision-making: staying focused on the ultimate goal, 2018. Available: https:// catalyst.nejm.org/shared-decision-making-patient-decision-aids/

13 Drug and Therapeutics Bulletin. An introduction to patient decision AIDS. BMJ 2013;347:f4147

14 Merchant FM, Dickert NW, Howard DH. Mandatory Shared Decision Making by the Centers for Medicare \& Medicaid Services for Cardiovascular Procedures and Other Tests. JAMA 2018;320:641.
15 Comprehensive Care for Joint Replacement Model [Internet]. Baltimore, MD: Center for Medicare and Medicaid Services, 2021. Available: https://innovation.cms.gov/innovation-models/cjr

16 Brook EM, Glerum KM, Higgins LD, et al. Implementing patientreported outcome measures in your practice: pearls and pitfalls. Am J Orthop 2017:46:273-8.

17 Forsberg HH, Nelson EC, Reid R, et al. Using patient-reported outcomes in routine practice: three novel use cases and implications. $J$ Ambul Care Manage 2015;38:188-95.

18 Harle CA, Listhaus A, Covarrubias CM, et al. Overcoming barriers to implementing patient-reported outcomes in an electronic health record: a case report. J Am Med Inform Assoc 2016;23:74-9.

19 Légaré F, Adekpedjou R, Stacey D, et al. Interventions for increasing the use of shared decision making by healthcare professionals. Cochrane Database Syst Rev 2018;2018.

20 Stacey D, Légaré F, Lewis K, et al. Decision AIDS for people facing health treatment or screening decisions. Cochrane Database Syst Rev 2017;2017.

21 Elwyn G, Durand MA, Song J, et al. A three-talk model for shared decision making: multistage consultation process. BMJ 2017;359:j4891.

22 Franklin P, Chenok K, Lavalee D, et al. Framework to guide the collection and use of patient-reported outcome measures in the learning healthcare system. EGEMS 2017;5:17

23 Development and Evaluation of Patient-Reported Outcome Score Visualization to Improve Their Utilization (PROVIZ) [Internet]. New York, NY: AHRQ Digital Healthcare Research: Informing Improvement in Care Quality, Safety, and Efficiency. Available: https://digital.ahrq. gov/ahrq-funded-projects/development-and-evaluation-patientreported-outcome-score-visualization-improve

24 Rheumatology Informatics System for Effectiveness PatientReported Outcome (RISE PRO) Dissemination Project (California) [Internet]. San Francisco, CA: AHRQ Digital Healthcare Research: Informing Improvement in Care Quality, Safety, and Efficiency. Available: https://digital.ahrq.gov/ahrq-funded-projects/ rheumatology-informatics-system-effectiveness-patient-reportedoutcome-rise-pro

25 Optimizing the Value of Patient-Reported Outcome Measures in Improving Care Delivery through Health Information Technology (Minnesota) [Internet]. Minneapolis, MN: AHRQ Digital Healthcare Research: Informing Improvement in Care Quality, Safety, and Efficiency. Available: https://digital.ahrq.gov/ahrq-funded-projects/ optimizing-value-patient-reported-outcome-measures-improvingcare-delivery

26 Curran GM, Bauer M, Mittman B, et al. Effectiveness-implementation hybrid designs: combining elements of clinical effectiveness and implementation research to enhance public health impact. Med Care 2012;50:217-26.

27 Jayakumar P, Moore MG, Furlough KA, et al. Comparison of an artificial Intelligence-Enabled patient decision aid vs educational material on decision quality, shared decision-making, patient experience, and functional outcomes in adults with knee osteoarthritis: a randomized clinical trial. JAMA Netw Open 2021; 4:e2037107.

28 Palinkas LA, Aarons GA, Horwitz S, et al. Mixed method designs in implementation research. Adm Policy Ment Health 2011;38:44-53.

29 Hurley DA, Murphy LC, Hayes D, et al. Using intervention mapping to develop a theory-driven, group-based complex intervention to support self-management of osteoarthritis and low back pain (SOLAS). Implement Sci 2016;11:56.

30 Hays RD, Bjorner JB, Revicki DA, et al. Development of physical and mental health summary scores from the patient-reported outcomes measurement information system (PROMIS) global items. Qual Life Res 2009;18:873-80.

31 Lyman S, Lee Y-Y, Franklin PD, et al. Validation of the KOOS, jr: a short-form knee arthroplasty outcomes survey. Clin Orthop Relat Res 2016;474:1461-71.

32 Sepucha KR, Stacey D, Clay CF, et al. Decision quality instrument for treatment of hip and knee osteoarthritis: a psychometric evaluation. BMC Musculoskelet Disord 2011;12:149.

33 Elwyn G, Barr PJ, Grande SW, et al. Developing collaborate: a fast and frugal patient-reported measure of shared decision making in clinical encounters. Patient Educ Couns 2013;93:102-7.

34 Linder SK, Swank PR, Vernon SW, et al. Validity of a low literacy version of the decisional conflict scale. Patient Educ Couns 2011;85:521-4

35 O'Connor AM. Validation of a decisional conflict scale. Med Decis Making 1995;15:25-30.

36 Brehaut JC, O'Connor AM, Wood TJ, et al. Validation of a decision regret scale. Med Decis Making 2003;23:281-92. 
37 Fogg B. A behavior model for persuasive design. Proceedings of the 4th International Conference on Persuasive Technology - Persuasive '09, Claremont, California: ACM Press, 2009:1.

38 Finley EP, Huynh AK, Farmer MM, et al. Periodic reflections: a method of guided discussions for documenting implementation phenomena. BMC Med Res Methodol 2018;18:153.

39 Fitzmaurice GM, Laird NM, Ware JH. Applied longitudinal analysis. 2nd ed. Hoboken, N.J: Wiley, 2011: 701.

40 Preisser JS, Lohman KK, Rathouz PJ. Performance of weighted estimating equations for longitudinal binary data with Drop-outs missing at random: weighted estimating equations for Drop-outs. Statist Med 2002;21:3035-54.

41 Malterud K, Siersma VD, Guassora AD. Sample size in qualitative interview studies: guided by information power. Qual Health Res 2016;26:1753-60.

42 Gale RC, Wu J, Erhardt T, et al. Comparison of rapid vs in-depth qualitative analytic methods from a process evaluation of academic detailing in the Veterans health administration. Implement Sci 2019;14:11.

43 Finley EP, Schneegans S, Tami C, et al. Implementing prescription drug monitoring and other clinical decision support for opioid risk mitigation in a military health care setting: a qualitative feasibility study. J Am Med Inform Assoc 2018;25:515-22.

44 Kroenke K, Spitzer RL, Williams JBW. The patient health Questionnaire-2: validity of a two-item depression screener. Med Care 2003;41:1284-92.

45 Kroenke K, Spitzer RL, Williams JB. The PHQ-9: validity of a brief depression severity measure. J Gen Intern Med 2001;16:606-13.

46 Skapinakis $P$. The 2-item generalized anxiety disorder scale had high sensitivity and specificity for detecting GAD in primary care. Evid Based Med 2007;12:149.

47 Spitzer RL, Kroenke K, Williams JBW, et al. A brief measure for assessing generalized anxiety disorder: the GAD-7. Arch Intern Med 2006;166:1092-7. 